\title{
Adenosine Sheds Light on the Relationship between Alcohol and Sleep
}

\author{
Corinde E. Wiers \\ Department of Psychiatry and Psychotherapy, Charité-Universitätsmedizin Berlin, 10117 Berlin, Germany, and Berlin School of Mind and Brain, \\ Humboldt-Universität zu Berlin, 10099 Berlin, Germany \\ Review of Clasadonte et al.
}

It has been extensively demonstrated that there is a strong association between sleep and alcohol consumption. While drinking small amounts of alcohol can help one falling asleep, chronic alcohol use often leads to reductions in the quality of sleep, and $\sim 50 \%$ of alcohol-dependent patients suffer from insomnia (Nam et al., 2012). However, the causal relationship between disturbed sleep and alcohol abuse and dependence is unclear. Wong et al. (2010) found that sleep problems in children were a strong marker of alcohol abuse later in life. Moreover, sleep disturbances have been shown to be a predictor of relapse in recovering alcohol-dependent patients, presumably since patients with sleep concerns are more likely to use alcohol to help them fall asleep (Nam et al., 2012).

The neurobiological mechanisms underlying the relationship between alcohol and sleep remain largely unexplored, but adenosine signaling may contribute. Altered signaling of adenosine in the brain is involved in the pathophysiology of both alcohol dependence and sleep disorders (Nam et al., 2012). In cell cultures, acute exposure to alcohol inhibits adenosine reuptake via the equilibrative nucleoside

Received March 29, 2014; revised April 25, 2014; accepted April 30, 2014. I thank Andrey Verendeev and Scott Stensland for proofreading. Correspondence should be addressed to Corinde E. Wiers, Berlin School of Mind and Brain, Humboldt-Universität zu Berlin, Unter den Linden 6, 10099 Berlin, Germany. E-mail: corinde.wiers@gmail.com.

DOI:10.1523/JNEUROSCI.1274-14.2014

Copyright $\odot 2014$ the authors $\quad 0270-6474 / 14 / 347733-02 \$ 15.00 / 0$ transporter type 1 (ENT1), leading to increased extracellular concentrations of adenosine. In contrast, chronic exposure to alcohol downregulates the expression of ENT1, so that alcohol-mediated inhibition of ENT1 is reduced and extracellular adenosine decreases (Nagy et al., 1990). Furthermore, in vivo rodent studies have shown that adenosine is a key player in the behavioral effects of alcohol, such as the promotion of sleep and the impairment of motor movements. For example, as a recent study by Sharma et al. (2014) demonstrates, the sleep-promoting effects of acute alcohol result from an adenosine A1 receptor-mediated inhibition of orexin neurons in the hypothalamus. These neurons are known to maintain wakefulness when activated, whereas a loss of orexin neurotransmission in the hypothalamus promotes sleep and can cause narcolepsy (Gerashchenko et al., 2001; Sharma et al., 2014). Moreover, there is increasing evidence that the motor-impairing effects of alcohol are mediated by A1 receptors in several brain areas, including the cerebellum, striatum, and cortex (Choi et al., 2004; Nam et al., 2012). Despite these findings, however, whether chronic sleep restriction alters adenosine signaling, and whether this influences the sensitivity to alcohol, has remained unexplored.

Clasadonte and colleagues (2014) have recentlyaddressed thesequestions. C57BL/ $6 \mathrm{~J}$ mice were forced to move on a treadmill for $4 \mathrm{~h}$ every $6 \mathrm{~h}$ for $3 \mathrm{~d}$ in a row, to chronically restrict their sleep. Control mice were not forced to run and hence could sleep normally. During the first day of sleep restriction, both hippocampal adenosine tone (i.e., adenosine-dependent inhibition of synaptic activity, measured by recording field EPSPs in hippocampal slices that were perfused with an A1 antagonist) and slow-wave activity (a measure of sleep quality, measured with EEG) increased. However, after $3 \mathrm{~d}$ of sleep restriction, Clasadonte et al. (2014) found the opposite: both these measures decreased. Reductions in adenosine tone lasted for 2 weeks and were likely due to a loss of source of extracellular adenosine rather than an increase in adenosine reuptake. That is, pharmacologically blocking ENT1 (which would inhibit adenosine reuptake) had no effect in sleep-restricted mice, suggesting that the active delivery of extracellular adenosine was affected. These findings indicate that chronic sleep restriction impairs sleep homeostasis and decreases adenosine tone.

Next, the authors investigated the behavioral consequences of chronic sleep restriction on alcohol sensitivity. Sleeprestricted and non-restricted mice were placed on a rotarod, a rotating cylinder, $1 \mathrm{~d}$ after chronic sleep restriction. There were no group differences in performance on the rotarod when the mice were in a drug-free state. However, after intoxicating the animals with alcohol, sleeprestricted mice performed better than control mice in that they showed lower latencies to fall and a faster recovery (50\% 
of the drug-free baseline performance). The same effects were found 2 and 4 weeks after sleep restriction and were not due to differences in alcohol metabolism. Therefore, the findings demonstrate that chronic sleep-restricted mice were less sensitive to the motor-impairing effects of alcohol, an effect that lasted at least a month after restriction. Interestingly, infusion of an $\mathrm{A} 1$ receptor antagonist into the brain resulted in better rotarod performances under the influence of alcohol, but in control mice only. Although indirectly, this suggests that sleep restriction affects the sensitivity to the motorimpairing effects of alcohol via alternations in adenosine signaling.

The findings of Clasadonte et al. (2014) are significant in that they provide evidence for a relationship between sleep restriction and alcohol sensitivity, mediated by adenosine signaling. How then is decreased sensitivity to alcohol related to alcohol consumption? Although Clasadonte et al. (2014) did not investigate this directly, evidence from previous mouse and human studies suggests that decreased sensitivity of motor behaviors to the impairing effects of alcohol leads to increases in alcohol consumption. First, Choi et al. (2004) showed that genetically modified mice missing ENT1 (ENT1-null mice) were not only less sensitive to the acute effects of alcohol on rotarod performances, but also drank more alcohol than wild-type mice. Since ENT1-null mice had reduced levels of adenosine and A1 receptors in the striatum, and the administration of an A1 agonist decreased drinking in mice, adenosine signaling may be directly involved in alcohol sensitivity and alcohol consumption. Second, human studies measuring ataxia on the body sway test, in which participants wear a harness that measures body movements, showed that lower intensity of both alcohol-induced body sway and subjective responses to intoxication at age 20 were potent predictors of alcohol abuse or dependence almost 10 years later (Schuckit, 1994). This relationship was found for both sons of alcoholics and controls without a family history of alcohol dependence. Therefore, reduced sensitivity to the motor-impairing effects of alcohol may lead to more positive experiences of alcohol, making it more likely to drink larger amounts. Moreover, higher levels of intake could lead to alcohol tolerance, in which higher doses of alcohol are needed to get similar effects, which further increases the risk of abusing alcohol or developing dependence (Schuckit, 1994).

The findings of Clasadonte et al. (2014) may have clinical importance. Specifically, if a reduced motor-impairing sensitivity to alcohol is a strong predictor of alcohol consumption (Schuckit, 1994; Choi et al., 2004) and this sensitivity is related to reduced adenosine levels, then drugs that increase adenosine A1 receptor signaling could potentially be potent in reducing alcohol consumption. However, both A1 receptor agonists and adenosine kinase inhibitors have negative side effects, making the process of drug development difficult (Nam et al., 2012). Additionally, where in the brain such an intervention could decrease alcohol sensitivity and alcohol consumption remains unknown. Clasadonte et al. (2014) researched adenosine signaling in the hippocampus of sleep-restricted mice, but it remains unclear whether the effects on alcohol sensitivity were mediated at the hippocampal level. In fact, it seems unlikely that the hippocampus is related to the motor-impairing effects of alcohol for two reasons. First, diminished sensitivity to the motor-impairing effects of alcohol and alcohol-intake have been associated with decreases in adenosine receptors in the cerebellum, striatum, and cortex (Choi et al., 2004; Nam et al., 2012), but not in the hippocampus. Second, neither alcohol nor an inhibitor of ENT1 in the rat hippocampus lead to increases in extracellular adenosine levels (Diao and Dunwiddie, 1996). This lack of response hence suggests that the sensitivity to alcohol is influenced by adenosine signaling in a different brain area than the hippocampus. Consistent with this, the A1 receptor antagonist that decreased sensitivity to alcohol in control mice in Clasadonte et al. (2014) was distributed globally in the brain and may have acted in different brain regions. Further studies are necessary to investigate where in the brain the effects of sleep-restriction on alcohol sensitivity take place.

Based on the results of Clasadonte et al. (2014), a noninvasive strategy to reduce alcohol sensitivity may be the promotion of sleep. Although more insight is needed into whether sleep restriction also decreases motor sway after alcohol intoxication in humans, the study of Clasadonte et al. (2014) is the beginning of exciting findings regarding the relationship between sleep and alcohol sensitivity and consumption.

\section{References}

Choi DS, Cascini MG, Mailliard W, Young H, Paredes P, McMahon T, Diamond I, Bonci A, Messing RO (2004) The type 1 equilibrative nucleoside transporter regulates ethanol intoxication and preference. Nat Neurosci 7:855-861. CrossRef Medline

Clasadonte J, McIver SR, Schmitt LI, Halassa MM, Haydon PG (2014) Chronic sleep restriction disrupts sleep homeostasis and behavioral sensitivity to alcohol by reducing the extracellular accumulation of adenosine. J Neurosci 34:1879-1891. CrossRef Medline

Diao L, Dunwiddie TV (1996) Interactions between ethanol, edogenous adenosine and adenosine uptake in hippocampal brain slices. J Pharmacol Exp Ther 278:542-546. Medline

Gerashchenko D, Kohls MD, Greco M, Waleh NS, Salin-Pascual R, Kilduff TS, Lappi DA, Shiromani PJ (2001) Hypocretin-2-saporin lesions of the lateral hypothalamus produce narcoleptic-like sleep behavior in the rat. J Neurosci 21:7273-7283. Medline

Nagy LE, Diamond I, Casso DJ, Franklin C, Gordon AS (1990) Ethanol increases extracellular adenosine by inhibiting adenosine uptake via the nucleoside transporter. J Biol Chem 265:1946-1951. Medline

Nam HW, McIver SR, Hinton DJ, Thakkar MM, Sari Y, Parkinson FE, Haydon PG, Choi DS (2012) Adenosine and glutamate signaling in neuron-glial interactions: implications in alcoholism and sleep disorders. Alcohol Clin Exp Res 36:1117-1125. CrossRef Medline

Schuckit MA (1994) Low level of response to alcohol as a predictor of future alcoholism. Am J Psychiatry 151:184-189. Medline

Sharma R, Sahota P, Thakkar MM (2014) Role of adenosine and the orexinergic perifornical hypothalamus in sleep-promoting effects of ethanol. Sleep 37:525-533. CrossRef Medline

Wong MM, Brower KJ, Nigg JT, Zucker RA (2010) Childhood sleep problems, response inhibition, and alcohol and drug outcomes in adolescence and young adulthood. Alcohol Clin Exp Res 34:1033-1044. CrossRef Medline 